\title{
On relative equilibria of mutually gravitating massive point and triangular rigid body
}

\author{
Vasily I. Nikonov \\ Lomonosov Moscow State University, Department of Mechanics and Mathematics \\ email: nikon_v@list.ru
}

\begin{abstract}
Planar motions of a triangular body and a massive point under the action of mutual Newtonian attraction are studied. For the first formulation the triangle is assumed to be composed of three massive points. For the second formulation it is constructed with three homogeneous rods. Some partial solutions are observed within the analysis of the geometry of mass distribution.

The investigation is motivated by the problem of motion of spacecrafts near asteroid-like celestial objects possessing irregular mass distribution. Comparison of dynamical effects for two types of mass distribution is another goal of the research.

Problems appearing because of irregularities in mass distributions have been known for a long time. Certain approaches to the description of motions under attraction as well as qualitative particulars of dynamics are discussed by Demin (1963), Burov \& Karapetyan (1995), Buchin \& Burov \& Troger (2008), Burov \& Guerman \& Sulikashvili (2010), Kholshevnikov \& Kuznetsov (2011), Beletsky \& Rodnikov (2011) and Scheeres (2012).
\end{abstract}

Keywords. celestial mechanics, generalized two-body problem, relative equilibria, stability, Poincaré bifurcation diagram, barycentric coordinates

Consider a motion of $\Delta P_{1} P_{2} P_{3}$ and the point mass $P$ in the fixed plane under the action of mutual attraction. The triangular object $\Delta P_{1} P_{2} P_{3}$ is assumed to be composed of masses $m_{1}, m_{2}$ and $m_{3}$ located at its vertices ("point triangle", $\mathrm{PT}$ ), or by homogeneous rods $P_{1} P_{2}, P_{2} P_{3}$ and $P_{1} P_{3}$ of masses $m_{3}, m_{1}$ and $m_{2}$ respectively ("wire triangle", WT). Let $P$ have mass $m$.

Denote $M=m_{1}+m_{2}+m_{3},\left|P_{1} P_{2}\right|=\ell_{3}>0(1,2,3)$, where $(1,2,3)$ is the cyclic permutation of indices. Let $C$ and $S$ be the centers of mass of the triangle and the whole system respectively, and $C_{f}$ be the point where the gravitational forces generated by the the triangle vanish. Then, the following statement is true.

Assertion. If $C=C_{f}$, then for any value of $m$ there exist steady motions of the triangle with an arbitrary angular velocity, such that $C=C_{f}=P$.

For PT the condition $C=C_{f}$ requires $C$ to be center of the circumscribed circle, i.e.

$m_{1}=\frac{\ell_{1}^{2}\left(-\ell_{1}^{2}+\ell_{2}^{2}+\ell_{3}^{2}\right)}{16 \mathbb{S}^{2}} \quad(1,2,3), \quad \mathbb{S}=\sqrt{p\left(p-\ell_{1}\right)\left(p-\ell_{2}\right)\left(p-\ell_{3}\right)}, \quad p=\frac{\ell_{1}+\ell_{2}+\ell_{3}}{2}$

For WT this condition is true if and only if $m_{1}=m_{2}=m_{3}$.

In case of PT, positivity of masses implies that all angles of the triangle are acute.

Using the Routh method (see Routh (1877)), one can investigate in general steady motions of the systems and their degrees of instability. If $\left(x_{1}, x_{2}, x_{3}\right)$ are barycentric coordinates $(\mathrm{BC})$ of the point $P$ with respect to the triangle $P_{1} P_{2} P_{3}$, then the Routh 

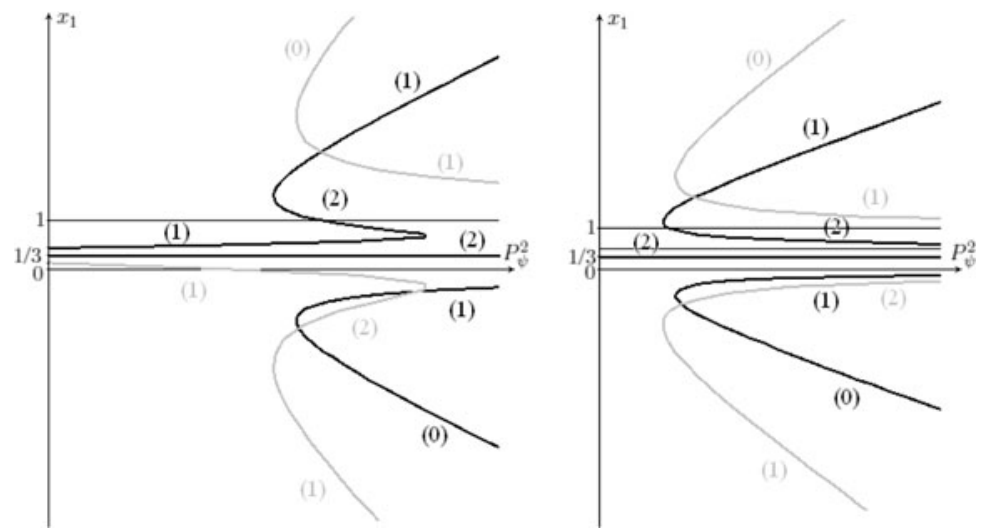

Figure 1. Poincare diagram: point and wire triangles

function leads to $W_{\lambda}=U+\lambda\left(x_{1}+x_{2}+x_{3}-1\right)$

$$
U= \begin{cases}\frac{1}{2} \frac{P_{\psi}^{2}}{J}-m G \sum_{i=1}^{3} \frac{m_{i}}{r_{i}} & \text { for } P T, \\ \frac{1}{2} \frac{P_{\psi}^{2}}{J}-m G \sum_{(1,2,3)} \frac{m_{1}}{\ell_{1}} \ln \frac{r_{2}+r_{3}+\ell_{1}}{r_{2}+r_{3}-\ell_{1}} & \text { for } W T,\end{cases}
$$

where $P_{\psi}$ is the constant of total angular momentum, $G$ is the gravitational constant, and $J$ is the moment of inertia of the whole system with respect to the axis perpendicular to the plane of motion and passing through the point $S$.

Steady configurations are determined as critical points of the function $W_{\lambda}\left(x_{1}, x_{2}, x_{3}, \lambda\right)$. Analyzing these equations one can easily discover families of steady motions, such that the point $P$ locates at one of the axes of dynamical symmetry of the triangle (see Nikonov (2014) for PT). Appropriate Poincaré bifurcation diagrams are drawn in Fig 1. There the figures near the curves express degrees of instability. In particular, the figure "0" corresponds to the stability of steady motion in the sense of Lyapunov. The Poincaré bifurcation diagram for PT drawn before in Nikonov (2014) is improved here.

\section{Acknowledgement}

The author acknowledges support of IAU and of RFBR (12-08-00637, 12-08-00591).

\section{References}

Aksenov, E. P., Grebenikov, E. A., \& Demin, V. G. 1963, Soviet Astron., 7, 276

Burov, A. A. \& Karapetyan, A. V. 1995, Mechanics of solids, 30, 14

Burov, A. A., Guerman, A. D., \& Sulikashvili, R. S. 2010, Dynamics of a Tetrahedral SatelliteGyrostat, AIP Conference Proceedings, ICNAAM 2010, 1281, 465

Kholshevnikov, K. V. \& Kuznetsov, E. D. 2011, Celest. Mech. Dyn. Astr., 109, 201

Scheeres, D. J. 2012, Orbital Motion in Strongly Perturbed Environmentss Berlin: Springer. $390 \mathrm{p}$.

Beletsky, V. \& Rodnikov, A. 2011, Proc. of 5th PhysCon, Leon, Spain.

Buchin, V., Burov, A., \& Troger, H. 2008, 6th EUROMECH Nonlinear Dynamics Conference. ENDC 2008, IPME RAS.

Routh, E. J. 1877, Treatise on the Stability of a Given State of Motion. L:MacMillan.

Nikonov, V. 2014, Moscow University Mechanics Bulletin, 69, 44 